\title{
PERFIL DERMATOGLIFICO DE JUGADORES PROFESIONALES DE FUTBOL DEL CLUB DEPORTIVO NUBLENSE DE LA CIUDAD DE CHILLAN
}

\section{DERMATOGLYPHIC PROFILE OF THE PROFESSIONAL SOCCER PLAYERS OF THE NUBLENSE SPORTS CLUB IN THE CITY OF CHILLAN}

\author{
Hernández Mosqueira, Claudio ${ }^{1,3}$, Hernández Vásquez, Dagoberto ${ }^{2} \&$ Fernandes Filho, José $^{3}$
}

${ }^{1}$ Director Carrera Pedagogía en Educación Física. Programa de Doctorado en Ciencias de la Motricidad Humana - PPGCMH. Universidad Pedro de Valdivia sede Chillan, Chile.

${ }^{2}$ Docente Carrera de Educación Física Universidad Pedro de Valdivia sede Chillan, Chile.

${ }^{3}$ Laboratorio de Biociencias del Movimiento Humano LABIMH / UFRJ - Rio de Janeiro, RJ / Brasil

HERNÁNDEZ M.C., HERNÁNDEZ V.D. \& FERNANDES F.J. (2013) Perfil Dermatoglifico de Jugadores Profesionales de Futbol del Club Deportivo Nublense de la Ciudad de Chillan. Mot. Hum. 14(1): 9-15.

\section{RESUMEN}

El objetivo de este estudio se centra en identificar el Perfil Dermatoglífico de los jugadores del plantel profesional de fútbol del Club Deportivo Nublense de la ciudad de Chillan. Es un estudio descriptivo de corte transversal. La muestra se compone de 20 jugadores profesionales de Fútbol del Club Deportivo Ñublense de Chillan, de sexo masculino con rangos promedios de edad de 24,8 \pm 4,92, Estatura 176,5 4,86 cm. Peso 74,5 $\pm 5,24 \mathrm{Kg}$. Para la evaluación de las Huellas Digitales se utilizó el protocolo de Dermatoglifía de Cummings \& Midlo, 1961. Para el análisis de los resultados se utilizó Estadística descriptiva, mínimos, máximos y desviación estándar. Para el Perfil Dermatoglífico fueron encontrados valores de diseño digital Arco (A) 2,0\%, Presilla (L) 55,0\%, Verticilo (W) 43,0\%. Índice de Deltas (D10) 14,6 \pm 2,50, Sumatoria Cantidad Total de Líneas (SQTL) 146,7 \pm 32,80. Al realizar la clasificación Dermatogíifica, se puede concluir que la muestra analizada se encuadra en clase $V$ con tendencia a ser maximizado los niveles somáticos funcionales de Coordinación y de Resistencia, minimizando los de Fuerza, Velocidad y Agilidad. Se demuestra la aplicabilidad práctica de la Dermatoglifía como marcador genético, en la optimización de estrategias de selección y orientación deportiva. Se recomiendan investigaciones sobre la correlación del estudio con variables físicas y en otros deportes.

Palabras Clave: Dermatoglifía, Genética, Fútbol, Rendimiento Atlético,

\begin{abstract}
The aim of this study focuses on identifying the dermatoglyphic profile of the professional soccer players of the Nublense sports club in the city of Chillan. Cross sectional descriptive study. The sample consists of 20 professional soccer players of the Nublense sports club in Chillan. Males with a mean age range of (24.8 \pm 4.92 years). Height (176.5 \pm $4.86 \mathrm{~cm})$. Weight $(74.5 \pm 5.24 \mathrm{Kg}$ ). In the assessment of the fingerprints, the dermatoglyphics, (Cummins \& Midlo, 1961). For the analysis of the results a descriptive statistics, was used, minimum, maximum and standard deviation. Results: In the dermatoglyphic profile the following digital design values were found, Arco (A) 2.0\%, Presilla $(L) 55 \%$, Verticilo (W) 43\%, and Delta Index (D10) (14.6 \pm 2.50). Sum Total Lines (LQTS) (146.7 \pm 32.80). In light of dermatoglyphic classification, it can be conclude that the sample analyzed falls in the class V category, with a tendency to maximize functional somatic levels of Coordination and Resistance and minimized levels of Speed, Agility and Strength. The practical applicability of dermatoglyphics as a genetic marker is demonstrated for optimizing selection strategies and sports orientation. Further investigations are recommended in order to study the correlation of physical variables and in other sports.
\end{abstract}

Keywords: Dermatoglyphics, Genetic, Soccer, High Performance 


\section{INTRODUCCIÓN}

En los últimos tiempos, la selección temprana de jóvenes talentos ha ido ganando importancia para las distintas modalidades deportivas, y mucho más en los deportes orientados al profesionalismo. En el fútbol, los clubes de primera división cuentan en su mayoría con equipos de categorías inferiores que constituyen la materia prima de su producto final y de la economía de los clubes. Por lo tanto es conveniente buscar mejorar esta selección y orientación de deportistas en edades tempranas. Para esta selección en la actualidad se utilizan Test Físicos, Test Técnico - Tácticos, Mediciones Corporales y Test Psicológicos (Llames, 1999; Patiño, Girón, Arango, 2007; Capetillo, 2010; Mercé et al., 2007; Soarez et al., 2012; González, 2008; Izquierdo et al., 2008; Jorquera et al., 2012).

El presente trabajo presenta el método Dermatoglífico como complemento a los ya mencionados. Según Fernandes Filho (Fernandes \& Abramova, 1997) las Huellas digitales son marcas genéticas, informativas y objetivas, que no dependen de la etnia y de la nacionalidad, pudiendo ser utilizadas, mundialmente, en la práctica, selección y la orientación deportiva. Además que al evaluar las Huellas digitales, permiten la opción más apropiada de especialización en el deporte, con la perspectiva de optimización del talento individual (Rolim \& Fernandes, 2007).

En los estudios de talentos deportivos es conveniente que las huellas dactilares pueden usarse como marcas genéticas en la identificación de las calidades físicas y que éstas son específicas para las modalidades diferentes, por consiguiente, no descubre el talento, indica sí, el potencial de desempeño deportivo, que es necesario para el éxito en cualquier modalidad deportiva, y más es un valioso instrumento en la orientación del talento (Fernandes, 2004). La Dermatoglifía - deriva del termino latín, dermo, significa "piel”; y del griego, glypha, "registro" - es un término, propuesto por Cummins y Midlo (Midlo, 1942). Fue planteado en la 42 ${ }^{\text {a }}$ Sesión Anual de la Asociación Americana de Anatomistas realizada en abril de 1926 (Dantas \& Fernandes, 2002) Para la interpretación de los diseños corresponde a la siguiente clasificación:

a) El nivel bajo del índice delta (D10), el aumento de los dibujos del Arco (A) y Presilha (L), y la disminución de dibujos de Verticilo (W), la disminución del sumatoria total de cantidad de líneas en todos los dedos (SQTL), todos son propios de las modalidades deportivas, con la potencia alta, y el tiempo corto de duración.

b) El nivel alto de D10, la falta del arco (A), el aumento de la porción del verticilo (W) el aumento SQTL, caracteriza a las modalidades deportivas de resistencia de velocidad, anterior. las modalidades de juegos, demuestre esta tendencia; Las modalidades de deportes de velocidad y de fuerza, ellos se insertan: en el campo de valores bajos de D10 y de SQTL;

c) En las modalidades, con el propiocepción compleja, en el campo de altos valores de D10;

d) En los grupos de deportes de resistencia, el D10 ocupa la posición intermedia. (Fernandes, 2004)

De acuerdo con Roquetti y Fernandes Filho, se propone la siguiente clasificación que se presenta en la tabla 1.

Tabla 1. Clasificación del conjunto de los Índices Dermatoglíficos y de los índices SomáticoFuncionales

\begin{tabular}{|c|c|c|c|c|}
\hline CLASES & D10 & SQTL & $\begin{array}{l}\text { No Desarrollado } \\
\text { Tiene que dar } \\
\text { mayores estímulos. } \\
\text { "Entrenar más" }\end{array}$ & $\begin{array}{c}\text { Desarrollado } \\
\text { Tiene que dar } \\
\text { estímulos para su } \\
\text { desarrollo }\end{array}$ \\
\hline I & 6,0 & 22 & $\begin{array}{c}\text { Coordinación, } \\
\text { Velocidad, Agilidad y } \\
\text { Resistencia. }\end{array}$ & Fuerza \\
\hline II & 9,1 & 86,2 & $\begin{array}{l}\text { Coordinación, } \\
\text { Agilidad y } \\
\text { Resistencia. }\end{array}$ & $\begin{array}{l}\text { Velocidad, Fuerza } \\
\text { y Fuerza Explosiva }\end{array}$ \\
\hline III & 11,1 & 119,1 & $\begin{array}{c}\text { Coordinación, } \\
\text { Resistencia, Agilidad. }\end{array}$ & $\begin{array}{c}\text { Velocidad y Fuerza } \\
\text { Explosiva }\end{array}$ \\
\hline IV & 14,1 & 139,6 & Velocidad y Fuerza & $\begin{array}{l}\text { Coordinación, } \\
\text { Resistencia y } \\
\text { Agilidad. }\end{array}$ \\
\hline $\mathbf{V}$ & 16,1 & 150,1 & $\begin{array}{c}\text { Fuerza, Velocidad y } \\
\text { Agilidad }\end{array}$ & $\begin{array}{l}\text { Coordinación y } \\
\text { Resistencia }\end{array}$ \\
\hline
\end{tabular}

La Dermatoglifía como método de selección ha demostrado su aplicación con una cantidad considerable de investigaciones con diferentes modalidades deportivas y con deportistas de Elite; siendo en Sudamérica, Brasil quien es pionera en esta materia (Castanhede, Dantas \& Fernandes, 2003; Fernandes, 2002; Medina \& Fernandes, 2002; Da Cunha, 2006; Souza \& Fernandes, 2006; Dos Santos, Dantas, Fernandes, 2008; Fonseca, 
Dantas, Fernandes, Fernandes, 2008; Anjos, Fernandes, Novaes, 2003; Mello \& Fernandes, 2004; Bastos, Dantas \& Fernandes, 2006; Fazolo, Cardoso, Tuche, Menezes, Teixeira, et al., 2005).

En la tabla 2 se presentan los resultados de distintas modalidades deportivas en equipos Brasileños y sus Valores para los Tipos de Diseño; SQTL y D10

Tabla 2. Tabla de Referencia de los Índices Dermatoglificos en Brasil en deportistas de Alto Rendimiento o Profesionales

\begin{tabular}{|c|c|c|c|c|c|c|}
\hline MODALIDAD & $\mathbf{N}$ & $\begin{array}{c}\mathrm{A} \\
(\%)\end{array}$ & $\begin{array}{c}\mathrm{L} \\
(\%)\end{array}$ & $\begin{array}{l}\mathrm{W} \\
(\%)\end{array}$ & D10 & SQTL \\
\hline Voleibol (2000) & 22 & 1,00 & 65,00 & 34,00 & 13,4 & 125,6 \\
\hline $\begin{array}{l}\text { Voleibol } \\
(1997)\end{array}$ & 28 & 0,70 & 53,20 & 46,10 & 14,5 & 133,8 \\
\hline $\begin{array}{l}\text { Voleibol } \\
\text { Femenino }\end{array}$ & 12 & 12,00 & 59,00 & 29,00 & 11,8 & 98,6 \\
\hline $\begin{array}{l}\text { Basquetbol } \\
\text { (1997) }\end{array}$ & 35 & 2,00 & 60,00 & 38,00 & 13,6 & 136,7 \\
\hline $\begin{array}{l}\text { Basquetbol } \\
\text { Masculino } \\
\text { Camp. }\end{array}$ & 12 & & & & & \\
\hline Panamericano & & 5,00 & 69,20 & 25,80 & 12,1 & 12,1 \\
\hline Karate (1997) & 7 & 0,00 & 45,70 & 54,30 & 15,4 & 159,7 \\
\hline Boxeo (1997) & 5 & 0,00 & 46,00 & 54,00 & 15,4 & 143,4 \\
\hline $\begin{array}{l}\text { Gimnasia } \\
\text { Olímpica }\end{array}$ & 25 & 6,40 & 62,80 & 30,80 & 12,4 & 97,8 \\
\hline $\begin{array}{l}\text { Hándbol } \\
\text { Femenino }\end{array}$ & 20 & 23,00 & 57,00 & 21,00 & 9,8 & 74 \\
\hline Triatlón & 10 & 6,00 & 6,50 & 29,00 & 12,3 & 118,6 \\
\hline Pilotos de Caza & 20 & 2,70 & 63,80 & 33,50 & 13,1 & 129,4 \\
\hline $\begin{array}{l}\text { Futbol de } \\
\text { Campo }\end{array}$ & 48 & 5,80 & 69,00 & 25,60 & 12 & 99,2 \\
\hline $\begin{array}{l}\text { Fustal } \\
\text { Masculino }\end{array}$ & 21 & 0,50 & 45,70 & 53,80 & 15,3 & 142,1 \\
\hline $\begin{array}{l}\text { Futbol Playa } \\
\text { Atletismo }\end{array}$ & 10 & 3,00 & 46,00 & 51,00 & 14,8 & 131,6 \\
\hline $\begin{array}{l}\text { Masculino } 100 \\
\text { mts } \\
\text { Atletismo }\end{array}$ & 10 & 14,30 & 62,90 & 22,90 & 10,9 & 93 \\
\hline $\begin{array}{l}\text { Femenino } 100 \\
\text { mts }\end{array}$ & 10 & 3,60 & 71,80 & 24,50 & 12,1 & 102,7 \\
\hline $\begin{array}{l}\text { Buceo de } \\
\text { Profundidad }\end{array}$ & 31 & 4,20 & 67,70 & 28,10 & 12,4 & 129 \\
\hline $\begin{array}{l}\text { Esgrima } \\
\text { Femenino }\end{array}$ & 8 & 11,00 & 68,00 & 21,00 & 11 & 80,5 \\
\hline Vela & 4 & 0,00 & 65,00 & 35,00 & 13,5 & 130,8 \\
\hline $\begin{array}{l}\text { Natación } \\
\text { Velocidad }\end{array}$ & 4 & 7,80 & 61,10 & 31,10 & 12,3 & 110 \\
\hline Natación Fondo & 6 & 1,70 & 41,70 & 56,70 & 15,5 & 153,8 \\
\hline
\end{tabular}

En Chile, La Federación de Fútbol de Chile (F.F.C.) fue fundada el 19 de junio de 1895, Es una de las federaciones nacionales más antiguas del mundo, después de las de Inglaterra y Argentina, siendo la segunda federación de América en antigüedad. Es una de las cuatro entidades fundadoras de la CONMEBOL en 1916. La Federación de Fútbol está asociada a la FIFA, a la
CONMEBOL, al Comité Olímpico Internacional y al Comité Olímpico de Chile. A la vez, es el organismo que rige al fútbol profesional y al fútbol amateur chileno. Además la federación es representada internacionalmente por la Selección de fútbol de Chile y la Selección femenina de fútbol de Chile en todas sus categorías.

La Federación de Fútbol de Chile es la encargada de supervisar a las siguientes asociaciones:

- Asociación Nacional de Fútbol Profesional (ANFP): Originalmente llamada Asociación Central de Fútbol (ACF). Conformada por los clubes profesionales de fútbol.

- Asociación Nacional de Fútbol Amateur de Chile (ANFA): Conformada por los clubes no profesionales de fútbol.

Dentro de este contexto surge la necesidad de conocer en más profundidad la modalidad en sus diversos aspectos. Por esto motivo este estudio considera su problema la necesidad, de conocer la modalidad en los aspectos Dermatoglíficos, procurando atender los deseos de la comunidad académica, así como de los profesionales que están envueltos con la modalidad.

El Objetivo del presente estudio fue Identificar el Perfil Dermatoglífico de los jugadores del plantel profesional de Fútbol del Club Deportivo Nublense de la ciudad de Chillan, campeonato de Apertura del año 2010.

\section{MATERIAL Y METODOS}

El presente estudio es de tipo descriptivo con un corte transversal, presenta una tipología de perfil y de delineamiento ex post-facto. De acuerdo con la propuesta, de Thomas \& Nelson, 2007.

\section{Muestra.}

Para este estudio la muestra utilizada se compuso por 20 Futbolistas Profesionales de Fútbol del Club Deportivo Ñublense de Chillan. La edad media del grupo fue de $24,8+/$ - 4,92 años, con un peso corporal de $74,50+/-5,24 \mathrm{~kg}$ y una talla de 176,5 $+/-4,84 \mathrm{~cm}$.

\section{Procedimientos.}

Los sujetos fueron informados de los objetivos del estudio y firmaron el término de participación consentida. Posteriormente, fueron dadas las recomendaciones referentes a los procedimientos regulares durante la recolección de los datos. En esta ocasión, los sujetos fueron orientados en 
cuanto a los procedimientos a ser realizados para la recolección de las Huellas digitales.

\section{Protocolo de Recolección de las Huellas Digitales (Dermatoglifia).}

Para el análisis de las Huellas Digitales, se utilizó el protocolo de Dermatoglifía de Cummins e Midlo, 1961. Para la toma de muestras se utilizó el Lector de Huellas Digitales Verifier ${ }^{\circledR} 300$ LC 2.0. La técnica utilizada para la toma de las Huellas, fue la de dedo rodado, que consiste en posicionar el dedo en el centro del lector y comenzar a rodar hacia el lado derecho y luego al izquierdo a la orden del evaluador. Después de realizada la recolección de las Huellas digitales, fueron hechos los procesamientos preliminares de lectura cuyo método padrón es el siguiente:

Determinar los tipos de diseños de los dedos de las manos.

Los tipos de diseño en las falanges distales de los dedos de las manos (Figura 1) son:

Arco "A" (diseño sin deltas). La característica principal es la ausencia de trirradios o deltas, y se compone de crestas que atraviesan, transversalmente, la almohada digital (Figura 1-A).

Presilha "L" - Diseño de un delta (posee un delta). Se trata de un diseño medio cerrado en que las crestas cutáneas comienzan de un extremo del dedo, se encorvan distalmente en relación al otro, sin acercarse a donde inician. (Figura 1-B);

Verticilo "W" - (Diseños de dos deltas). Se trata de una figura cerrada, en que las líneas centrales se concentran en torno del núcleo delo diseño (FIGURA 1-C).

\section{Figura 1}

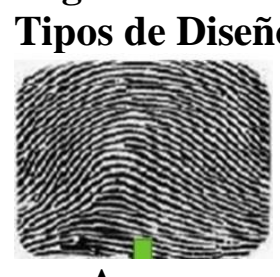

Arco

1a

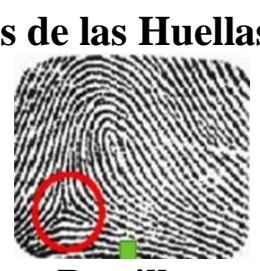

Presilha

$1 \mathrm{~b}$

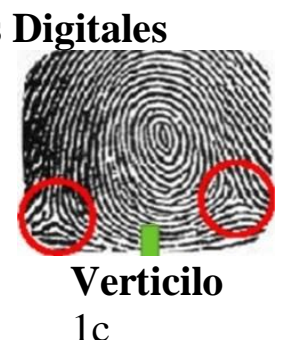

$1 \mathrm{c}$

\section{Cantidad de Líneas $(Q L)$}

La cantidad de líneas de las crestas cutáneas, dentro del diseño, y se cuentan de acuerdo a la línea que une el delta y el centro del dibujo, sin ser tomadas en cuenta la primera y última línea de las crestas.

En este punto, fueron calculados los índices padronizados fundamentales de las Huellas digitales que son:

a) La cantidad de los diseños para los diez dedos de las manos.

b) La sumatoria de las líneas (QL) en cada dedo de las manos.;

c) Cálculo del índice Delta (D10) Que se obtiene de la suma de deltas de todos los diseños, de modo que el valor relativo de Arco (A) es siempre 0 (debido a la ausencia de delta); de cada Presilha $(\mathrm{L})=1$ (un delta); de cada Verticilo $(\mathrm{W})=2($ dos deltas), finalmente para determinar el D10 se aplica la siguiente formula $\mathrm{D} 10=\Sigma \mathrm{L}+2 \Sigma \mathrm{W}$;

d) La sumatoria total de las líneas (SQTL) de los diez dedos de las manos.

\section{Estadística}

Para el análisis de los resultados se utilizó Estadística descriptiva, mínimos, máximos y desviación estándar.

\section{RESULTADOS}

A continuación se presentan los valores medios y sus derivados de las variables Edad, Peso y Estatura del plantel profesional de Fútbol del Club Deportivo Nublense de Chillan en la Tabla 3.

Tabla 3. Valores Medios y sus derivados para Edad, Peso y Estatura

\begin{tabular}{llll}
\hline & $\begin{array}{l}\text { Edad } \\
\text { (años) }\end{array}$ & $\begin{array}{l}\text { Peso } \\
(\mathbf{k g})\end{array}$ & $\begin{array}{l}\text { Estatura } \\
(\mathbf{c m})\end{array}$ \\
\hline $\mathrm{N}$ & 20 & 20 & 20 \\
Promedio & 24,8 & 74,5 & 176,5 \\
$\mathrm{Ds}$ & 4,92 & 5,24 & 4,86 \\
Mínimo & 17,8 & 60,0 & 168,5 \\
Máximo & 34,6 & 83,7 & 184,0 \\
\hline
\end{tabular}

Los valores medios y sus derivados, referentes a los tipos de diseño, A, L, W son presentados, en la Tabla 4. En esta se puede observar que los diseños tipo $\operatorname{arco}(\mathrm{A})=2,0 \% \pm 0,52$, presilha $(\mathrm{L})=55,0 \%$ $\pm 2,24$, verticilo $(\mathrm{W})=43,0 \% \pm 2,34$.

Tabla 4 Valores Medios y sus derivados para los Tipos de Diseño 


\begin{tabular}{llll}
\hline & A & L & W \\
\hline $\mathrm{N}$ & 20 & 20 & 20 \\
Promedio & $2,0 \%$ & $55,0 \%$ & $43,0 \%$ \\
Mínimo & 0 & 2 & 0 \\
Máximo & 2 & 10 & 8 \\
Fuente: Autores de este estudio & &
\end{tabular}

Los valores medios y sus derivados, referentes a los valores de SQTL y D10; son presentados, en la Tabla 5. En esta se puede observar la sumatoria de cantidad total de líneas $(\mathrm{SQTL})=146,7 \pm 37,42$, y del índice delta $(\mathrm{D} 10)=14,6 \pm 2,55$.

Tabla 5 Valores Medios y sus derivados para SQTL y D10.

\begin{tabular}{lll}
\hline & D10 & SQTL \\
\hline $\mathrm{N}$ & 20 & 20 \\
Promedio & 14,6 & 146,7 \\
Ds & 2,55 & 37,42 \\
Mínimo & 9 & 63 \\
Máximo & 18 & 215 \\
\hline
\end{tabular}

Finalmente con la intención de ser ofrecida una visualización, sobre el perfil total, la figura 2 expone todos los ítems observados, por medio de sus medidas normalizadas.

\section{Figura 2}
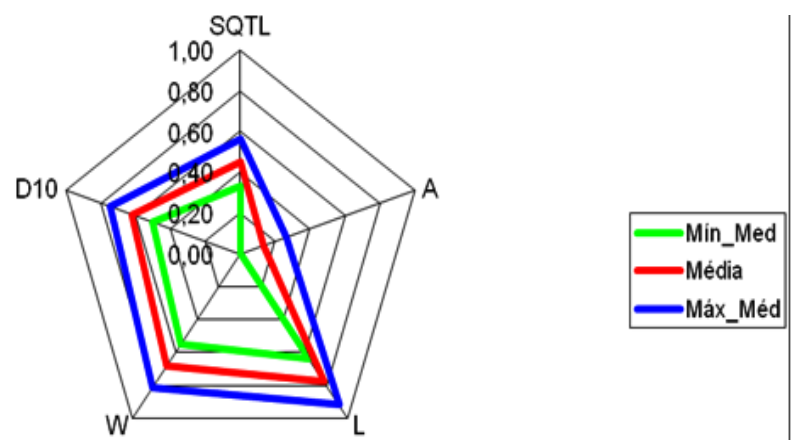

Finalmente en la Figura 3 se observa el Grafico de Radar de Fernandes Filho, que presenta el Perfil Total del Grupo.

Figura 3 - Gráfico radar con Perfil Total Valores Normalizados

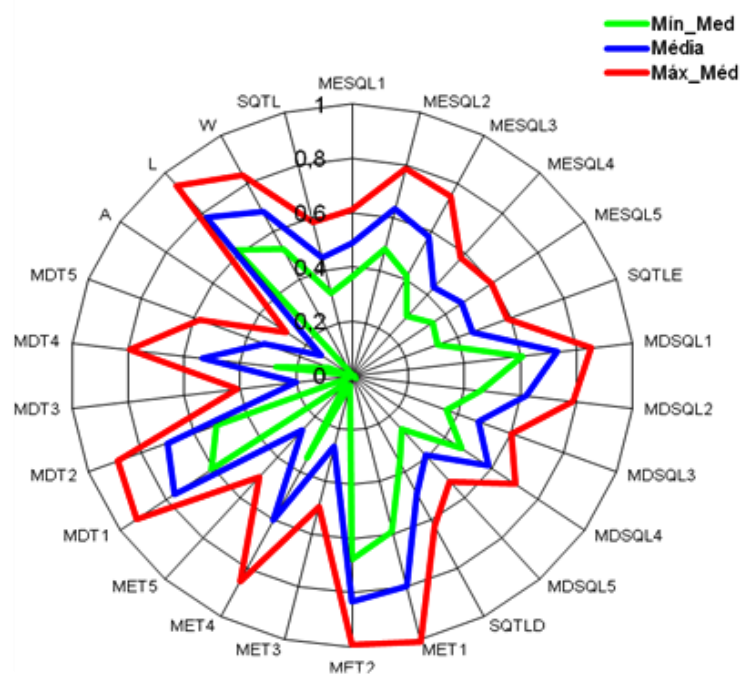

\section{DISCUSIÓN}

Para llegar al alto rendimiento es necesario la interacción de varios factores, entre los que se encuentran las características cineantropométricas, la condición física y las habilidades motoras. Siendo el Fútbol caracterizado por ser un ejercicio físico discontinuo, intermitente $y$ de gran intensidad, en el que se alternan carreras y períodos de reposo con saltos o carrera continua de baja intensidad; obteniéndose energía para todas estas acciones a partir de las tres vías metabólicas, aunque no está determinada con precisión la contribución de cada una de ellas (Sanuy, Peirau, Biosca, Perdrix, 1995). Según Fernandes Filho (Mello \& Fernandes, 2004), el alto índice de D10 y SQTL, caracterizan las modalidades deportivas que necesitan Fuerza, Velocidad y Agilidad y que tienen desarrollada la Coordinación y Resistencia.

Sobre el tipo de diseño y de la cantidad de líneas encontrados en los dedos de las dos manos, se concluye que la baja presencia de Arco y la predominancia de Presilha sobre Verticilo, son características propias de los jugadores de Fútbol de alto nivel, con valores similares a los de Castanhede (Castanhede, et al., 2003), realizado en Futbolistas de alto rendimiento de Rio de Janeiro - Brasil. Donde evaluaron el Perfil Dermatoglífico y somatotípico de 48 atletas de fútbol de campo masculino de alto rendimiento en Rio de Janeiro - Brasil, encontramos diferencias en la cantidad de diseños de A $(5,8 \%), \mathrm{L}(69,0 \%)$, W $(25,6 \%)$. Donde existe una gran correlación de presilhas ligadas a la Fuerza Explosiva y de Verticilos ligadas a la coordinación, características 
presentes en este tipo de deporte (Dantas \& Fernandes, 2002).

Finalizando, el perfil Dermatoglífico del Equipo Profesional de Fútbol del Club Deportivo Nublense de Chillan; este se encuadra en la clase V de clasificación de Índices Somático Funcionales de acuerdo a Roquetti y Fernandes Filho (2004), De esta forma se presentan maximizados los niveles somático- funcionales de Coordinación y de Resistencia, minimizando los de Fuerza, Velocidad y Agilidad.

\section{CONCLUSIONES}

La presente investigación busco caracterizar las marcas informativas, objetivas y de orientación de la selección deportiva por medio de los índices cualitativos y cuantitativos que representan las Huellas digitales. Los Futbolistas Profesionales del club deportivo Nublense de Chillan analizados presentan una tendencia de anulación del diseño digital $\mathrm{A}$, con $\mathrm{L}$ y $\mathrm{W}$ en una posición cuantitativa de predominancia.

Estas informaciones, obtenidas a través de la Dermatoglifía, son inestimables herramientas para la planificación de los entrenamientos y la aplicación en el desarrollo de cualidades físicas básicas propuestas para el deporte.

\section{Recomendaciones.}

Finalmente la identificación de este perfil deportivo puede ser ampliada a través de la verificación de las diversas cualidades físicas, logrando, de esta forma, la optimización de posibles aptitudes o deficiencias a ser superadas. Al identificar el perfil de estos deportistas, a futuro será posible establecer, de manera científica los criterios para el desarrollo de los puntos sensibles, de interés deportivos y de la selección de los futuros Futbolistas Profesionales del club deportivo Nublense de Chillan. Por este motivo se recomienda realizar más estudios, con un universo mayor para la identificación del perfil Dermatoglífico y de variables físicas, composición corporal y somatotipo de Futbolistas Profesionales Chilenos e incluir a otros deportes.

\section{REFERENCIAS BIBLIOGRAFICAS}

Alves Costa C, Gomes Silva H, Mendes Da Silva H, De Souza Capistrano R. Perfil Dermatoglífico e Características Somatomotoras de Atletas Jóvenes de Futsal. X - Livro de Memorias do VI Congresso Científico Norte-nordeste CONAFF.

Anjos M, Fernandes Filho J, Novaes J. (2003). Características somatotípicas, dermatoglífi cas e fi siológicas do atleta de triatlo. Fitness \& Performance Journal. 2(1):4957.

Bastos F, Dantas P, Fernandes Filho J. (2006). Dermatoglifia, somatotipo e qualidades físicas básicas no basquetebol: estudo comparativo entre as posições. Motricidade 2(1): 3252 .

Capetillo Velásquez R. (2010). La identificación de talentos deportivos para deportes de habilidad abierta. Una visión socio deportiva. Lúdica pedagóg. 2(15):148-155.

Castanhede A, Dantas, P, Fernandes Filho J. (2003). Perfil Dermatoglífico e somatotípico, de atletas de fútbol de campo masculino, do alto rendimento no Rio de Janeiro - Brasil. Fitness \& Performance Journal, 2(4): 234-239.

Da Cunha Júnior A, Trevisan Schneider A, Da Cunha A, Silva Dantas P. (2006). Características dermatoglíficas, somatotípicas, psicológicas e fisiológicas da seleção brasileira feminina adulta de handebol. Fitness \& Performance Journal. 5(2):81-86.

Dantas P, Fernandes Filho J. (2002). Identifi cação dos perfis genético, de aptidão física e somatotípico que caracterizam atletas masculinos de alto rendimento, participantes do futsal adulto no Brasil. Fitness \& Performance Journal. 1(1): 28-36.

Dos Santos L, Dantas P, Fernandes filho J. (2008). Características genotípicas e fenotípicas em atletas velocistas. Motri. [online]. 4(1):48-56.

Fazolo E, Cardoso P, Tuche W, Menezes I, Teixeira M, Portal M, et al. (2005). A Dermatoglifía e a Somatotipologia No Alto Rendimiento Do Becah Soccer - Selecao Brasileira. Revista de Educacao Física. 130:45-51

Fernandes Filho J. Dermatoglifía un Instrumento de Prescripción en el Deporte. FIEP Boletín, 2004.

Fernandes Filho J, Abramova T. (1997). A utilização de índices Dermatoglificos na seleção de talentos esportivos. Treinamento Desportivo. 2(1):41-46.

Fonseca C, Dantas P, Fernandes P, Fernandes Filho J. (2008). Perfil Dermatoglífico, somatotípico e da força explosiva de atletas da seleção brasileira de voleibol feminino. Fit Perf J. 7(1):35-40.

González De los Reyes Y. (2008). Validez, Fiabilidad y especificidad de las Pruebas de agilidad. rev.udcaactual.divulg.cient. 11(2): 31-39. 
Izquierdo JM, Zarzuela R, Sedano S, De Benito A, Salgado I. (2008). Estudio Comparativo de Factores Antropométricos y Físico - Técnicos en Jóvenes Futbolistas de Elite de Ambos sexos, en Función de la Posición Habitual de Juego. Actas V Congreso Asociación Española de Ciencias del Deporte.

João A, Fernandes Filho J. (2002). Identificação do perfil genético, somatotípico e psicológico das atletas brasileiras de ginástica olímpica feminina de alta qualifi cação esportiva. Fitness \& Performance Journal. 1(2):12-19.

Jorquera Aguilera C, Rodríguez Rodríguez F, Torrealba Viera I, Barraza Gómez F. (2012). Composición Corporal y Somatotipo de Futbolistas Chilenos Juveniles Sub 16 y Sub 17. Int. J. Morphol. 30(1): 247-252.

Llames Lavandera R. (1999). Selección de Jóvenes Deportistas en Fútbol. Revista de Psicología del Deporte. 8 (2): 249-257.

Medina M, Fernandes Filho J. (2002). Identificação dos perfi s genético e somatotípico que caracterizam atletas de voleibol masculino adulto de alto rendimento no Brasil. Fitness \& Performance Journal. 1(4):12-19.

Mello M, Fernandes Filho J. (2004). The dermatoglyphic, somatotypical and body composition profile of high level female judo athletes. Fitness \& Performance Journal. 3(6): 340-348.

4.- Mercé Cervera J, Mundina Gómez J, García Herreros R, Yagüe Cabezón J M, González Moreno L. (2007). Estudio de un modelo para los procesos cognitivos en jugadores de fútbol de edades comprendidas entre 8 y 12 años. http://www.efdeportes.com/ Revista Digital - Buenos Aires. 11(105).

Midlo C, Cummins H. (1942). Palmar and plantar dermatoglyphics in primates. The American Anatomical Memoirs. The Wistar Institute of Anatomy and Biology, Philadelphia. 20: 111-198.

Patiño Fernández W, Girón Muñoz V, Arango Valencia A. (2007). Parámetros Técnico - Tácticos para la Selección de Jugadores de Fútbol en las Categorías sub 13 - 16 con miras al Alto Rendimiento. Indeportes Antioquia - Liga Antioqueña de Fútbol.

Rolim Filho N, Fernandes Filho J. (2007). Identificación do Perfil Dermatoglífico e Somatotipico de Pentatletas Modernos Brasileiros de Alto Rendimiento. Revista de Educacao Física. 139:29-39

Sanuy Bescós X, Peirau Terés X, Biosca Estelo P, Perdrix Ecequiel R. (1995). Fisiología del Fútbol: Revisión Bibliográfica. Revista Apunts, Educación Física y Deportes. (42): 55-60.

Soarez H, Fragoso I, Massuc L, Barrigas C. (2012). Impacto de la maduración y de los puestos específicos en la condición física en jóvenes Futbolistas. Apunts Med Esport. 47(174):73-81
Souza Menezes L, Fernandes Filho J. (2006). Identificação e comparação das características dermatoglíficas, somatotípicas e qualidades físicas básicas de atletas de GRD de diferentes níveis de qualificação esportiva . Fitness \& Performance Journal. 5(6):393-401.

\section{Dirigir Correspondencia a:}

Claudio Marcelo Hernández Mosqueira

Universidad Pedro de Valdivia - Panamericana

Norte $\mathrm{N}^{\circ} 3651$ Chillan - Chile.

Email: chernandez@upv.cl

RECIBIDO: 12-05-2013

ACEPTADO: 13-06-2013 